\title{
Coarsening kinetics of fine-scale microstructures in deformed materials
}

\author{
Yu, Tianbo; Hansen, Niels
}

Published in:

Acta Materialia

Link to article, DOI:

10.1016/j.actamat.2016.08.032

Publication date:

2016

Document Version

Peer reviewed version

Link back to DTU Orbit

Citation (APA):

Yu, T., \& Hansen, N. (2016). Coarsening kinetics of fine-scale microstructures in deformed materials. Acta Materialia, 120, 40-45. https://doi.org/10.1016/j.actamat.2016.08.032

\section{General rights}

Copyright and moral rights for the publications made accessible in the public portal are retained by the authors and/or other copyright owners and it is a condition of accessing publications that users recognise and abide by the legal requirements associated with these rights.

- Users may download and print one copy of any publication from the public portal for the purpose of private study or research.

- You may not further distribute the material or use it for any profit-making activity or commercial gain

- You may freely distribute the URL identifying the publication in the public portal

If you believe that this document breaches copyright please contact us providing details, and we will remove access to the work immediately and investigate your claim 
Coarsening kinetics of fine-scale microstructures in deformed materials

Tianbo $\mathrm{Yu}^{*}$ and Niels Hansen

Section for Materials Science and Advanced Characterization, Department of Wind Energy, Risø

Campus, Technical University of Denmark, DK-4000, Roskilde, Denmark

Tianbo $\mathrm{Yu}^{*}$ :

Tel: $\quad$ (45) 46775825

Fax: (45) 46775758

Email: tiyu@dtu.dk

Address: Building 228, DTU Risø Campus, DK-4000, Roskilde, Denmark

Niels Hansen: $\quad$ Email: niha@dtu.dk

*Corresponding author. 


\begin{abstract}
:
In this work we consider three representative continuous coarsening processes, namely subgrain growth in deformed subgrain structures, triple junction motion in deformed lamellar structures, and grain growth in deformed nanocrystalline structures, spanning a large range in structural scale and driving force. We propose a unified coarsening model, which is based on recovery kinetics and allows the apparent activation energy to change during coarsening. The model is successfully applied to the three coarsening processes in different materials of different structural morphology and scale, showing that the apparent activation energy increases during coarsening, which is verified by direct calculation. The increase in the apparent activation energy dominates the coarsening kinetics and leads to a significant decrease in the coarsening rate as coarsening proceeds. This suggests that a conventional grain growth model is not applicable in an analysis of coarsening of nanostructured materials. Our analysis also shows that an initial low thermal stability of nanostructured materials is inherently related to their large boundary area per unit volume and their high content of stored energy, reducing the activation barrier for structural coarsening.
\end{abstract}

\title{
Keywords:
}

Coarsening kinetics; Deformation structure; Activation energy; Annealing; Nanocrystalline materials 


\section{Introduction}

In the design and development of strong metallic materials, plastic deformation to high and ultrahigh strains has been under rapid development in the last 20 years [1-6]. The materials owe their high strength to a very fine microstructure subdivided by boundaries on the submicrometer and nanometer scale. The energy stored in the structure is high, providing a large driving force for recovery and boundary migration [7]. These materials therefore lack thermal stability and may easily lose strength when applied or even during storage [8]. In design and development it is therefore mandatory to understand and model softening processes in these strong materials, and the present study focuses on boundary migration.

Recovery kinetics in deformed metals has been explored extensively in the literature [9-14], and recently a thorough study of recovery in Al cold rolled to medium and high strains has been carried out $[8,15-18]$. These studies show that the apparent activation energy increased continuously as recovery proceeds towards recrystallization $[13,15,18]$. Based on these studies, the current work widens the scope to model the general coarsening behavior of metallic materials after plastic deformation to medium-to-ultrahigh strains, where dislocations are preferentially stored in different types of boundaries, whose spacing varies on a length scale from a few micrometers to a few tens of nanometers.

When metallic materials of medium-to-high stacking fault energy are plastically deformed to low-to-medium strains, a cell-block structure typically forms, containing cell-blocks subdivided by cell boundaries $[4,19]$. At this strain range, the majority of deformation induced boundaries are low angle boundaries $\left(<15^{\circ}\right)$. During recovery, the boundaries become sharper and the structure may become coarser. This structure, especially after recovery to some extent, is conventionally known as a subgrain structure, where subgrain growth is an important recovery mechanism [12,20,21]. Deformation, for example by cold rolling, accumulative roll bonding and high pressure torsion, to medium-to-high 
strains typically leads to a finely spaced lamellar structure, containing extended lamellar boundaries parallel to each other and interconnecting dislocation boundaries in-between $[4,17]$. The lamellar boundaries are a combination of low angle and high angle boundaries $\left(>15^{\circ}\right)$ and the interconnecting boundaries are predominantly low angle boundaries. Further deformation to extreme strains, for example by ball milling, leads to an extreme grain refinement and an equiaxed deformed nanocrystalline structure, where high angle boundaries dominate. To cover a structural scale from the micrometer to the nanometer range, three representative well-characterized microstructures were chosen (Fig. 1).

(i) A subgrain structure: Al-0.05 wt.\% Si single crystal channel die compressed by $70 \%$ at room temperature [22].

(ii) A lamellar structure: commercial purity Al with a main chemical composition of 99.5Al$0.25 \mathrm{Fe}-0.15 \mathrm{Si}$ (wt.\%) deformed by cold rolling to a true strain of $5.5[18,23]$;

(iii) A nanocrystalline structure: high purity $\mathrm{W}$ powder ball milled at room temperature in $\mathrm{Ar}$ atmosphere [24].
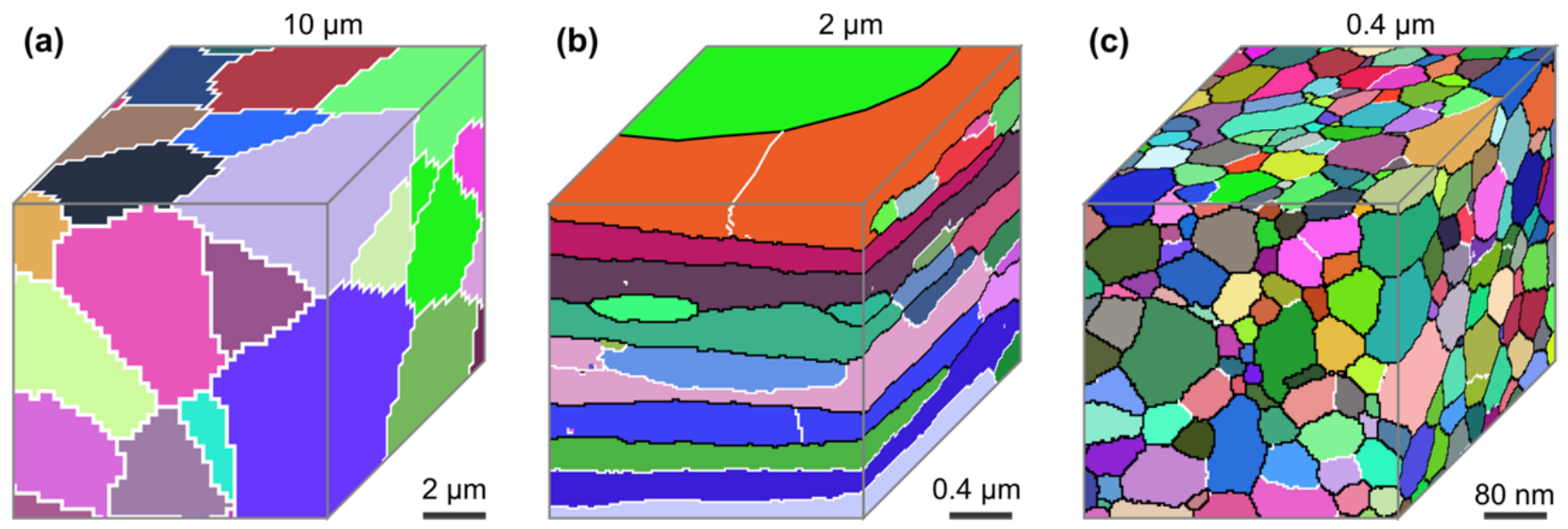
Fig. 1. Illustrations of three deformation structures of different length scales. (a) A subgrain structure; (b) a lamellar structure; (c) a nanocrystalline structure. White and black lines represent low and high angle boundaries, respectively. Different colors represent different grains/subgrains of different crystallographic orientations. The stored energy in the three structures spans almost two orders of magnitude.

\section{Model derivation}

The driving force for recovery and boundary migration results from the stored energy in the deformed materials. In our previous analysis of recovery kinetics, the remaining fraction of stored energy during annealing was determined by hardness indentation measurements $[15,16]$. In the present study, we estimate the stored energy by exclusively considering the energy stored in deformation induced boundaries since other contributions are comparatively small $[25,26]$. These boundaries have energies depending on their misorientation angles, and the boundary energy quickly approaches a constant with increasing misorientation angle according to the Read-Shockley equation [27]. During continuous coarsening of a deformation structure preceding recrystallization, the average misorientation angle typically changes marginally, and in the present work we take the average boundary energy as a constant during coarsening. Consequently, the stored energy $\left(\mathrm{J} / \mathrm{m}^{3}\right)$ can be simplified as $[20,26]$ $P=\gamma S_{V}$

where $\gamma$ is the average boundary energy $\left(\mathrm{J} / \mathrm{m}^{2}\right)$ and $S_{V}$ is the boundary area per unit volume $\left(\mathrm{m}^{-1}\right)$, which can be expressed as

$$
S_{V}=\frac{\alpha}{D}
$$


where $\alpha$ is a geometrical constant depending on the morphology and $D$ is a characteristic size parameter: (i) the subgrain size in a subgrain structure, (ii) the lamellar boundary spacing in a lamellar structure, and (iii) the grain size in a nanocrystalline structure. The morphology of a deformation structure may change slightly during continuous coarsening, but for simplicity we ignore this small change, i.e $\alpha$ is fixed. Therefore as a first approximation, the stored energy $P$ is reciprocally related to the characteristic size parameter $D$.

In order to account for a near logarithmic dependence of recovery kinetics, Kuhlmann [9] first suggested an increasing apparent activation energy during recovery. Later, Borelius and coworkers [10] suggested a similar rate equation for recovery, considering the zero recovery rate at the end

$\frac{d P}{d t}=-K_{0} P \exp \left(-\frac{Q_{0}-\beta P}{R T}\right)$

where $t$ is the annealing time, $T$ is the annealing temperature, $R$ is the gas constant, $Q_{0}$ is the activation energy at the end of recovery, and the three fitting parameters $K_{0}, Q_{0}$ and $\beta$ are associated with the operative recovery mechanisms. Eq. (3) may be recognized as a first-order reaction rate equation with an apparent activation energy depending linearly on the extent of recovery already occurred. The apparent activation energy is a key parameter in our study, and in the following the word apparent will be omitted for simplicity.

Combining Eqs. (1) to (3) gives the following expression

$$
\frac{d D}{d t}=k_{1} D \exp \left(\frac{k_{2}}{D T}\right)
$$

where $k_{1}$ is temperature dependent and $k_{2}$ is a constant, written as

$$
k_{1}=K_{0} \exp \left(-\frac{Q_{0}}{R T}\right)
$$


$k_{2}=\frac{\alpha \beta \gamma}{R}$

Eq. (4) can be solved with the aid of exponential integrals, resulting in the following relationship $\left(D=D_{0}\right.$ at $\left.t=0\right)$

$E i\left(-\frac{k_{2}}{D T}\right)-E i\left(-\frac{k_{2}}{D_{0} T}\right)=-k_{1} t$

where $E_{\mathrm{i}}()$ is the exponential integral of the quantity inside the bracket.

Eq. (7) describes the continuous coarsening of a deformation structure during isothermal annealing. At one annealing temperature in order to estimate the model parameters $k_{1}$ and $k_{2}$, coarsening data, i.e. $(t, D)$ pairs, are inserted into Eq. (7) and a curve of $k_{1}$ vs $k_{2}$ is calculated for each $(t$, $D$ ) pair. A maximum convergence point $\left(k_{2}, k_{1}\right)$ is then determined manually by superimposing $k_{1}$ vs $k_{2}$ curves of all annealing times at that temperature. The model thus gives a fitting of the isothermal coarsening kinetics. When such a procedure is carried out for other temperatures, different convergence points $\left(k_{2}, k_{1}\right)$ can be obtained, but the estimated temperature independent constant $k_{2}$ may vary. Therefore an important subsequent procedure is to use a single average $k_{2}$ for all temperatures to re-fit the coarsening data. Such a collective fitting can reduce the fitting error significantly, especially for estimating the activation energy $Q_{0}$ based on Eq. (5). Subsequently the activation energy at any stage of coarsening can be obtained as

$Q_{a p p}=Q_{0}-\frac{k_{2} R}{D}$

To ensure a satisfactory accuracy, typically three to four annealing temperatures are required, in combination with four to five annealing times at each temperature.

\section{Model application}




\subsection{Case I - Coarsening of a subgrain structure}

Huang and Humphreys [22] reported significant subgrain growth in Al-0.05\% Si single crystal of $\{110\}<001>$ orientation channel die compressed by $70 \%$ at room temperature $\left(D_{0}=0.9 \mu \mathrm{m}\right)$. The isothermal kinetics of continuous coarsening at five different temperatures measured by electron backscatter diffraction (EBSD) is shown in Fig. 2a. Based on Eq. (7), the convergence point $\left(k_{2}, k_{1}\right)$ for each annealing temperature is determined. Such initial fittings lead to an average value of $k_{2}=3.3 \times 10^{-3}$ $\mathrm{m} \cdot \mathrm{K}$ and correspondingly adjusted convergence points $\left(k_{2}, k_{1}\right)$ at different temperatures. The coarsening curves based on such collective fittings are drawn in Fig. 2a, showing a good agreement with experimental data over the whole range. 

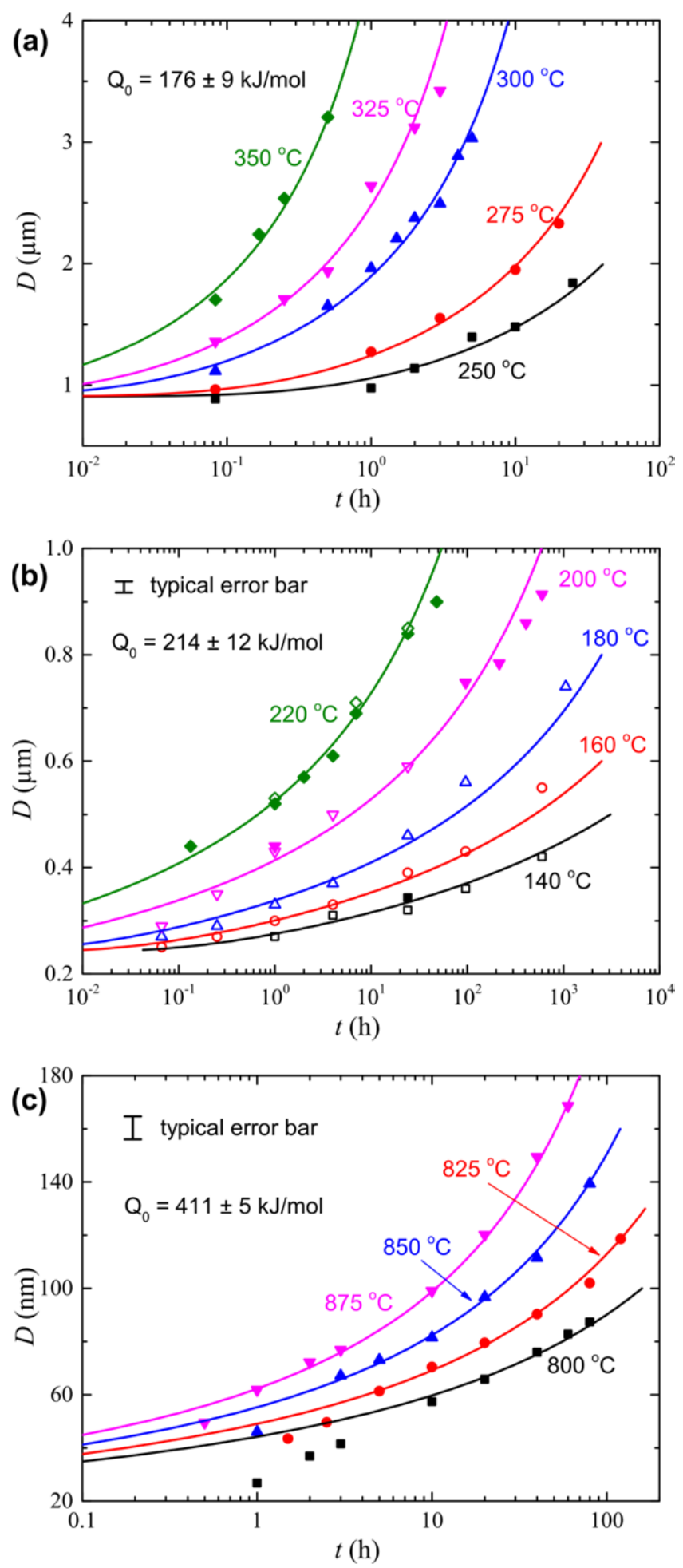
Fig. 2. Coarsening data in the literature fitted by the current model. (a) Isothermal subgrain growth kinetics of Al- $0.05 \%$ Si single crystal channel die compressed by $70 \%$ at room temperature [22]. (b) EBSD (solid symbols) and ECC (open symbols) data for the average lamellar boundary spacing during continuous coarsening in commercial purity Al cold rolled to a true strain of 5.5 [18]. (c) Crystallite size evolution during annealing of nanocrystalline high purity $\mathrm{W}$ powder produced by ball milling at room temperature in Ar atmosphere [24].

\subsection{Case II - Coarsening of a lamellar structure}

During annealing of heavily deformed commercial purity $\mathrm{Al}$ of lamellar structure, migration of triple junctions, each formed by three lamellar boundaries, led to a significant increase of the lamellar boundary spacing prior to nucleation of recrystallization $[17,28]$. In a recent study [18], the structural coarsening by triple junction motion in heavily cold-rolled ( $99.6 \%$ thickness reduction) commercial purity aluminum AA1050 $\left(D_{0}=0.24 \mu \mathrm{m}\right)$ was measured by EBSD and electron channeling contrast (ECC) imaging as shown in Fig. 2b. Annealing was carried out at five temperatures covering a time span from 4 minutes to 44 days, providing an ideal data set to test the current model. Based on Eq. (7), an average value of $k_{2}=1.87 \times 10^{-3} \mathrm{~m} \cdot \mathrm{K}$ can be obtained and the corresponding coarsening curves by collective fittings are drawn in Fig. 2b, showing a good agreement with the experimental data over a time span over four orders of magnitude.

The temperature dependence of $k_{1}$ determined by collective fitting is shown in Fig. 3a, and it follows that $Q_{0}=214 \pm 12 \mathrm{~kJ} / \mathrm{mol}$ according to Eq. (5). Based on Eq. (8), the dependence of the activation energy on the boundary spacing can also be calculated, for example $Q_{a p p}=149 \mathrm{~kJ} / \mathrm{mol}$ at $D_{0}$ $=0.24 \mu \mathrm{m}$. The activation energy increases rapidly at the beginning but slowly at later stages. By combining Eqs. (7) and (8), one can also derive the time dependence of the activation energy during 
annealing at different temperatures (Fig. 3b). The activation energy increases approximately logarithmically with the annealing time, and at a given annealing time the activation energy increases with increasing annealing temperature.
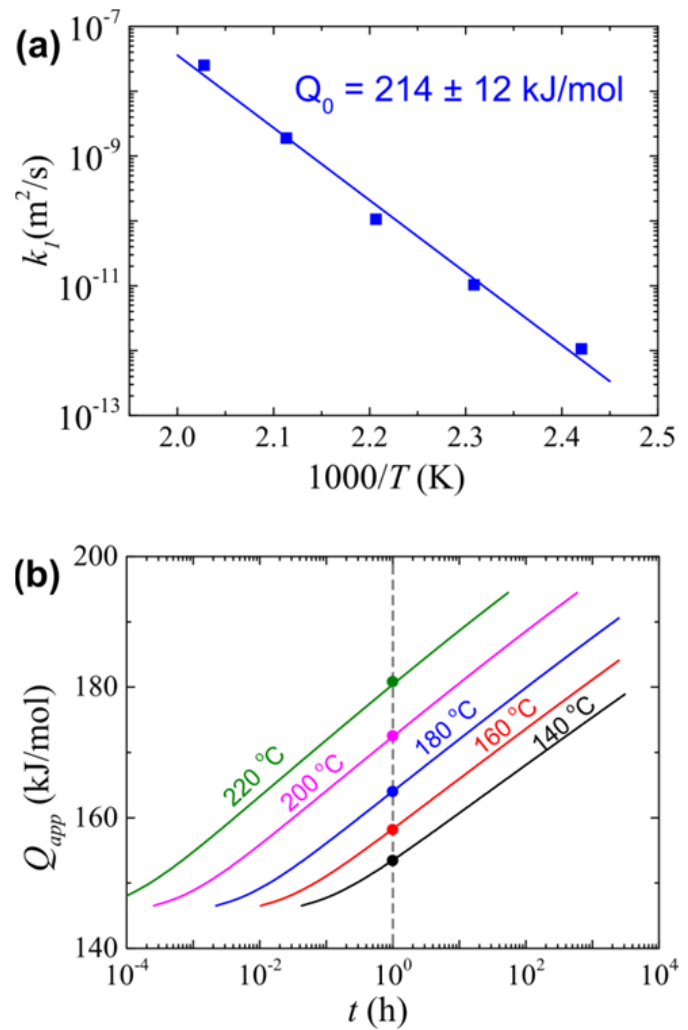

Fig. 3. Determination of the activation energy for Case II. (a) Temperature dependence of the fitting parameter $k_{1}$. The error in activation energy is calculated based on the standard error of linear least squares fitting. (b) Variation of the activation energy during annealing at five different temperatures, showing very different activation energies during isochronal annealing.

\subsection{Case III - Coarsening of a nanocrystalline structure}


In a recent work by Hegedus and coworkers [24], high purity W powder (particle size $<10 \mu \mathrm{m}$ ) was ball milled up to $4.5 \mathrm{~h}$, creating an equiaxed nanoscale structure with an average crystallite size of $D_{0}=20 \mathrm{~nm}$. The structural coarsening during annealing in vacuum was measured by in situ X-ray diffraction, and a number of data points are shown in Fig. 2c. An analysis similar to the above two cases leads to $k_{2}=3.7 \times 10^{-4} \mathrm{~m} \cdot \mathrm{K}$, and the collectively fitted curves are shown in Fig. $2 \mathrm{c}$. The estimated activation energy increases from $257 \mathrm{~kJ} / \mathrm{mol}$ at $D_{0}=20 \mathrm{~nm}$ to $396 \mathrm{~kJ} / \mathrm{mol}$ at $D=200 \mathrm{~nm}$. The structural coarsening is close to logarithmic, and no saturation is observed.

The fitting is acceptable except for the initial stage, where the crystallite size is less than $50 \mathrm{~nm}$. For example at $800^{\circ} \mathrm{C}$, the structure coarsened more in the second hour than in the first hour. Such data cannot be fitted together with the normal coarsening behavior at later stages, and this abnormality indicates that other factors may influence the coarsening kinetics in the initial stage; in this case it is probably due to the remaining $\mathrm{C}$ and $\mathrm{O}$, whose concentration decreased during annealing in vacuum [24]. Therefore this peculiar coarsening stage cannot be covered by the current model.

\section{Discussion}

\subsection{Activation energy}

The present coarsening model is based on recovery kinetics of deformed metals, and the key assumption is the linear dependence of the activation energy on the stored energy (driving force) $[9,10]$. This assumption appears rational and it gives good fittings with experimental data as shown in Fig. 2. In the following, the activation energies obtained from the current model will be compared to those from direct calculation based on the experimental data.

To calculate the activation energy, one has to assume the same coarsening mechanisms at different annealing temperatures, i.e. the same activation energy at a given grain size independent of 
temperature. Moreover, as an approximation, the activation energy is taken to be constant in a small coarsening window. These assumptions, together with the Arrhenius equation, enable a stepwise analysis of the activation energy as demonstrated recently [18]. As shown in Fig. 2, the structural coarsening is close to logarithmic, and therefore each small coarsening window may be extended to the beginning of coarsening and such an extension only increases the annealing time slightly. As a result, one may draw horizontal lines in Fig. 2, and then calculate the activation energy by comparing the annealing times (from interpolation of experimental data) at different temperatures based on the following equation

$\ln t=c+\frac{Q}{R T}$

where both $c$ and activation energy $Q$ are grain size dependent fitting parameters. Such a procedure is analogous to that for analyzing recrystallization kinetics [20]. However, the calculated activation energy corresponds to the final stage of each coarsening window, instead of the average value for the annealing period extended to the beginning of coarsening.

Based on Fig. 2 and Eq. (9), the directly calculated activation energies for the three examples are shown in Fig. 4 as discrete data points, which agree with the current modelling results shown as smooth curves. For coarsening of the Al- $0.05 \% \mathrm{Si}$ subgrain structure, the estimated activation energy is consistent with lattice diffusion of $\mathrm{Si}$ in $\mathrm{Al}$, whose activation energy is in the range of $129-154 \mathrm{~kJ} / \mathrm{mol}$ [29], indicating that solute drag can be a rate controlling mechanism. For coarsening of the commercial purity Al lamellar structure, the activation energies are consistent with diffusion of Fe in the bulk of $\mathrm{Al}$, where the activation energy is reported to be in the range of $183-259 \mathrm{~kJ} / \mathrm{mol}[30,31]$, also pointing to an important effect of solute drag. Moreover, the increase in the activation energy may be related to the removal of dislocations and a large fraction of boundaries during coarsening, which may not only 
reduce the contribution from short circuit diffusion but also increase the solute concentration in the remaining boundaries and thus enhance solute drag [32]. For coarsening of the $\mathrm{W}$ nanocrystalline structure, the activation energy is close to that for grain boundary diffusion of $\mathrm{W}, 381 \mathrm{~kJ} / \mathrm{mol}[33]$, suggesting that diffusion of $\mathrm{W}$ atoms along the boundary and across the boundary may require similar activation energies, and also that solute drag may not be important due to both the low concentration of substitutional solutes and the very fine structure. The agreement between the modelled activation energies and the directly calculated ones is illustrated in Fig. 4.

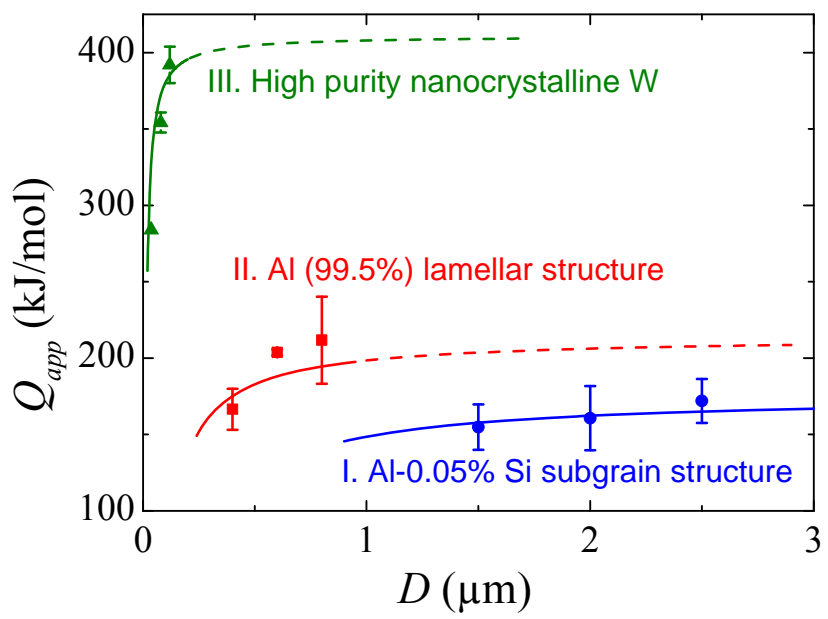

Fig. 4. Increase of the activation energy during coarsening of three deformation structures. The smooth curves obtained from the current model agree with the results (discrete points) directly calculated based on Eq. (9). The error bars are estimated according to the standard error of least squares fittings.

When the activation energy is known, the model is useful in predicting coarsening kinetics at other annealing times and temperatures. For example for Case II, the model predicts rapid coarsening within the first minute and slow coarsening afterwards during annealing at $300{ }^{\circ} \mathrm{C}$ (Fig. 5). The lamellar boundary spacing after 1 and 3 minutes are 0.7 and $0.85 \mu \mathrm{m}$, respectively, in good agreement 
with the experimental data reported by Mishin and coworkers [23]. When the boundary spacing is correctly predicted, the strength of the material can be subsequently calculated based on the Hall-Petch relationship.

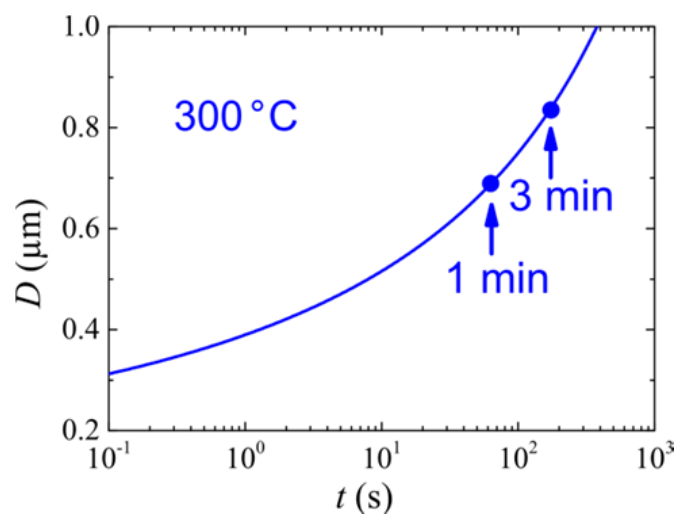

Fig. 5. Predicted coarsening kinetics of heavily cold-rolled $\mathrm{Al}$ during annealing at $300{ }^{\circ} \mathrm{C}$.

An increase in the activation energy during coarsening leads to a significant decrease in the coarsening rate, e.g. an increase by $10 \mathrm{~kJ} / \mathrm{mol}$ can typically reduce the coarsening rate by an order of magnitude. Therefore the model is robust and insensitive to other assumptions and simplifications. For example, the simplification of a reciprocal dependence between the stored energy $P$ and the size parameter $D$ only introduces small errors, typically less than $20 \%$, due to the presence of interior dislocations and variations of boundary energy and morphology. However, in some specially designed alloys, where the boundary energy changes significantly due to segregation, the variation of the boundary energy may have to be considered [34].

\subsection{Scale effect}


A decrease in the size parameter $D$ leads to an increase in the boundary area per unit volume, which provides a higher driving force for coarsening and a larger contribution from boundary diffusion. If solutes are present, an increase in the boundary area per unit volume will also reduce the concentration of solutes in boundaries. The change in boundary area is small when the grain size is above a couple of micrometers but becomes large when the grain size is in the submicrometer to the nanometer regime (Fig. 6). Since $Q_{a p p}=Q_{0}-\beta \gamma S_{V}$, the dramatic variation of the boundary area per unit volume in the finescale regime (left part of Fig. 6) explains the rapid increase of the activation energy shown in Fig. 4. Therefore coarsening of a fine-scale deformation microstructure is significantly different from conventional grain growth in a coarse-grained structure. For the latter process, the kinetics is well described by the following equation $[20,35]$

$D^{n}-D_{0}^{n}=k_{0} t \exp \left(-\frac{Q}{R T}\right)$

where $n$ is the growth exponent and $k_{0}$ is a kinetics constant.

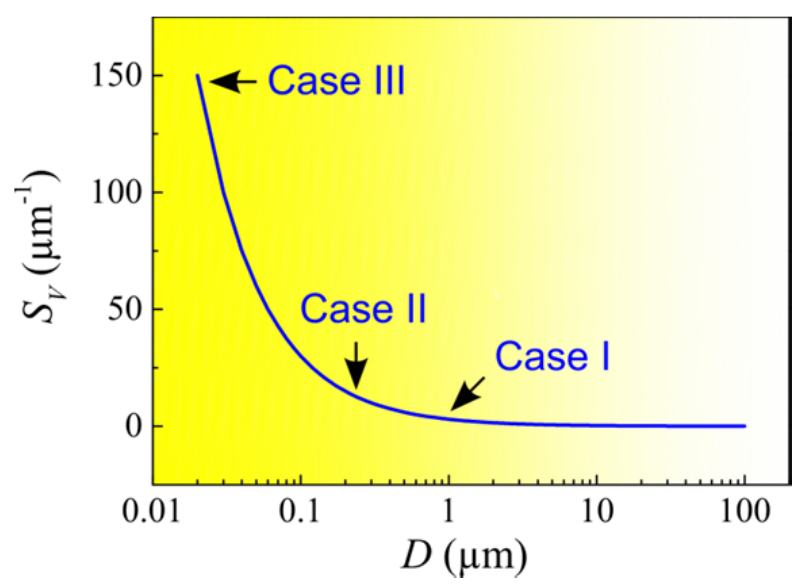


Fig. 6. Variation of the boundary area per unit volume $S_{V}$ with the size parameter $D$ (from $20 \mathrm{~nm}$ to 100 $\mu \mathrm{m}$ ) in a deformation structure, based on Eq. (2) with $\alpha=3$. The starting points of the three examples are indicated.

In the analyses of coarsening kinetics of fine-scale deformation microstructures, Eq. (10) has been frequently applied in the past [36]. When Eq. (10) with an assumption of a constant activation energy is used, a good fitting may be obtained at each annealing temperature, but the fitted growth exponent $n$ typically varies with temperature, and $n$ is much larger than the ideal number of 2 at low annealing temperatures $[18,22]$. If one disregards the large variation in the growth exponent and assigns the ideal growth exponent $n=2$ for all temperatures, then a good linear fitting may be achieved in a $\ln \left(D^{2}-D_{0}^{2}\right)$ vs $1 / T$ plot based on isochronal annealing data. However, such a procedure typically leads to a very low activation energy, inconsistent with diffusion mechanisms.

Based on the current work, it is clear from Fig. $3 \mathrm{~b}$ that during isochronal annealing the activation energy is very different at different annealing temperatures. With a higher temperature in a given annealing time, coarsening proceeds to a later (slower) stage associated with a higher activation energy, and therefore the coarsening rate may appear only weakly dependent on the temperature during isochronal annealing. Underestimation of the activation energy (i.e. underestimation of the temperature dependence) is therefore expected when Eq. (10) is used, although overestimation is also possible if a large growth exponent $n$ is chosen to exaggerate the difference in grain size. Therefore Eq. (7), instead of Eq. (10), may be used to better analyze continuous coarsening of fine-scale deformation microstructures, not only for the determination of activation energy and understanding of rate controlling mechanisms but also for the prediction of coarsening kinetics in materials where the parameters $k_{1}$ and $k_{2}$ have been determined experimentally. 


\section{Conclusion}

In the current work, the recovery kinetics first suggested by Kuhlmann [9] and later modified by Borelius and coworkers [10] has been generalized to a general coarsening description of fine-scale deformation structures. The model considers an important feature that the activation energy changes during coarsening, and it has been successfully applied to three representative examples, namely subgrain growth in a deformed subgrain structure, triple junction motion in a heavily deformed lamellar structure and grain growth in a ball-milled nanocrystalline structure. These coarsening processes occurred in structures of different morphology and scale, and modelling results show that the activation energies increase during coarsening, leading to a significant decrease in the coarsening rate with increasing time. The increase in the activation energy is related to the decrease in the boundary area per unit volume, which leads to a lower driving force and a smaller contribution from boundary diffusion. This coarsening model is essential for the design of nanocrystalline and nanostructured materials through thermomechanical processing, and it paves the way for the future development and application of these novel ultrastrong materials.

\section{Acknowledgements}

Professors D. Juul Jensen, A. Godfrey and X. Huang are thanked for helpful discussions. This research did not receive any specific grant from funding agencies in the public, commercial, or not-for-profit sectors.

\section{References}

[1] K. Lu, Stabilizing nanostructures in metals using grain and twin boundary architectures, Nat. 
Rev. Mater. (2016) 16019. doi:10.1038/natrevmats.2016.19.

[2] C.C. Koch, Structural nanocrystalline materials: an overview, J. Mater. Sci. 42 (2007) 14031414. doi:10.1007/s10853-006-0609-3.

[3] Y. Estrin, A. Vinogradov, Extreme grain refinement by severe plastic deformation: A wealth of challenging science, Acta Mater. 61 (2013) 782-817. doi:10.1016/j.actamat.2012.10.038.

[4] N. Hansen, C. Barlow, Plastic deformation of metals and alloys, in: D.E. Laughlin, K. Hono (Eds.), Phys. Metall., 5th ed., Elsevier, Amsterdam, 2014: pp. 1681-1764.

[5] R.Z. Valiev, R.K. Islamgaliev, I.V. Alexandrov, Bulk nanostructured materials from severe plastic deformation, Prog. Mater. Sci. 45 (2000) 103-189. doi:10.1016/S0079-6425(99)00007-9.

[6] Y. Saito, H. Utsunomiya, N. Tsuji, T. Sakai, Novel ultra-high straining process for bulk materials-development of the accumulative roll-bonding (ARB) process, Acta Mater. 47 (1999) $579-583$.

[7] J.H. Driver, Stability of nanostructured metals and alloys, Scr. Mater. 51 (2004) 819-823. doi:10.1016/j.scriptamat.2004.05.014.

[8] T. Yu, N. Hansen, X. Huang, Recovery mechanisms in nanostructured aluminium, Philos. Mag. 92 (2012) 4056-4074. doi:10.1080/14786435.2012.704418.

[9] D. Kuhlmann, Zur theorie der nachwirkungserscheinungen, Zeitschrift Fur Phys. 124 (1948) 468-481. doi:10.1007/BF01668885.

[10] G. Borelius, S. Berglund, S. Sjoberg, Measurements on the evolution of heat during the recovery of cold-worked metals, Ark. Fys. 6 (1952) 143-149.

[11] J.T. Michalak, H.W. Paxton, Some recovery characteristics of zone-melted iron, TMS-AIME. 221 (1961) 850-857.

[12] R. Sandstrom, Subgrain growth occurring by boundary migration, Acta Metall. 25 (1977) 905911. doi:10.1016/0001-6160(77)90177-8.

[13] T. Furu, R. Ørsund, E. Nes, Subgrain growth in heavily deformed aluminum-experimental investigation and modeling treatment, Acta Metall. Mater. 43 (1995) 2209-2232. doi:10.1016/0956-7151(94)00410-2.

[14] E. Nes, Recovery revisited, Acta Metall. Mater. 43 (1995) 2189-2207.

[15] R.A. Vandermeer, N. Hansen, Recovery kinetics of nanostructured aluminum: Model and experiment, Acta Mater. 56 (2008) 5719-5727. doi:10.1016/j.actamat.2008.07.038 ER.

[16] T. Yu, N. Hansen, X. Huang, G. Winther, Recovery kinetics of commercial purity aluminium after large strain, in: J.C. Grivel, N. Hansen, X. Huang, D.J. Jensen, O. V Mishin, S.F. Nielsen, W. Pantleon, H. Toftegaard, G. Winther, T. Yu (Eds.), Proc. 30th Risø Int. Symp. Mater. Sci., Risø DTU, Roskilde, Denmark, 2009: pp. 393-400.

[17] T. Yu, N. Hansen, X. Huang, Recovery by triple junction motion in aluminium deformed to ultrahigh strains, Proc. R. Soc. A. 467 (2011) 3039-3065. doi:10.1098/rspa.2011.0097.

[18] T. Yu, N. Hansen, X. Huang, Linking recovery and recrystallization through triple junction 
motion in aluminum cold rolled to a large strain, Acta Mater. 61 (2013) 6577-6586. doi:10.1016/j.actamat.2013.07.040.

[19] Q. Liu, D. Juul Jensen, N. Hansen, Effect of grain orientation on deformation structure in coldrolled polycrystalline aluminium, Acta Mater. 46 (1998) 5819-5838.

[20] F.J. Humphreys, M. Hatherly, Recrystallization and related annealing phenomena, 2nd ed., Pergamon Press, Oxford, 2004.

[21] C.J.E. Smith, I.L. Dillamore, Subgrain growth in high-purity iron, Met. Sci. 4 (1970) 161-167.

[22] Y. Huang, F.J. Humphreys, Subgrain growth and low angle boundary mobility in aluminium crystals of orientation $\{110\}<001>$, Acta Mater. 48 (2000) 2017-2030.

[23] O.V. Mishin, A. Godfrey, D. Juul Jensen, N. Hansen, Recovery and recrystallization in commercial purity aluminum cold rolled to an ultrahigh strain, Acta Mater. 61 (2013) 53545364. doi:10.1016/j.actamat.2013.05.024.

[24] Z. Hegedüs, S.R. Meka, E.J. Mittemeijer, Crystallite growth in nanocrystalline tungsten; rate determining mechanism and the role of contaminations, Acta Mater. 105 (2016) 232-243. doi:10.1016/j.actamat.2015.12.012.

[25] T. Knudsen, W.Q. Cao, A. Godfrey, Q. Liu, N. Hansen, Stored energy in nickel cold rolled to large strains, measured by calorimetry and evaluated from the microstructure, Metall. Mater. Trans. A. 39 (2008) 430-440.

[26] A. Godfrey, Q. Liu, Stored energy and structure in top-down processed nanostructured metals, Scr. Mater. 60 (2009) 1050-1055. doi:10.1016/j.scriptamat.2009.02.014.

[27] W.T. Read, W. Shockley, Dislocation models of crystal grain boundaries, Phys. Rev. 78 (1950) 275-289. doi:10.1103/PhysRev.78.275.

[28] T. Yu, D.A. Hughes, N. Hansen, X. Huang, In situ observation of triple junction motion during recovery of heavily deformed aluminum, Acta Mater. 86 (2015) 269-278. doi:10.1016/j.actamat.2014.12.014.

[29] L.F. Mondolfo, Aluminum alloys: structure and properties, Butterworths, London, 1976.

[30] G.M. Hood, Diffusion of iron in aluminium, Philos. Mag. 21 (1970) 305-328. doi:10.1080/14786437008238419.

[31] D.L. Beke, I. Godney, I.A. Szabo, G. Erdelyi, F.J. Kedves, On the diffusion of Fe-59 into aluminium and Al Mn solid solutions, Philos. Mag. A. 55 (1987) 425-443.

[32] D.A. Molodov, U. Czubayko, G. Gottstein, L.S. Shvindlerman, On the effect of purity and orientation on grain boundary motion, Acta Mater. 46 (1998) 553-564. doi:10.1016/S13596454(97)00277-2.

[33] I. Kaur, W. Gust, Handbook of grain and interphase boundary diffusion data, Ziegler Press, Stuttgart, 1989.

[34] Z. Chen, F. Liu, X.Q. Yang, C.J. Shen, A thermokinetic description of nanoscale grain growth: Analysis of the activation energy effect, Acta Mater. 60 (2012) 4833-4844. doi:10.1016/j.actamat.2012.05.029. 
[35] J.E. Burke, D. Turnbull, Recrystallization and grain growth, Prog. Met. Phys. 3 (1952) 220-292. doi:10.1016/0502-8205(52)90009-9.

[36] H. Asgharzadeh, H.J. McQueen, Grain growth and stabilisation of nanostructured aluminium at high temperatures: review, Mater. Sci. Technol. 31 (2015) 1016-1034. doi:10.1179/1743284714Y.0000000706.

\section{Graphic Abstract}
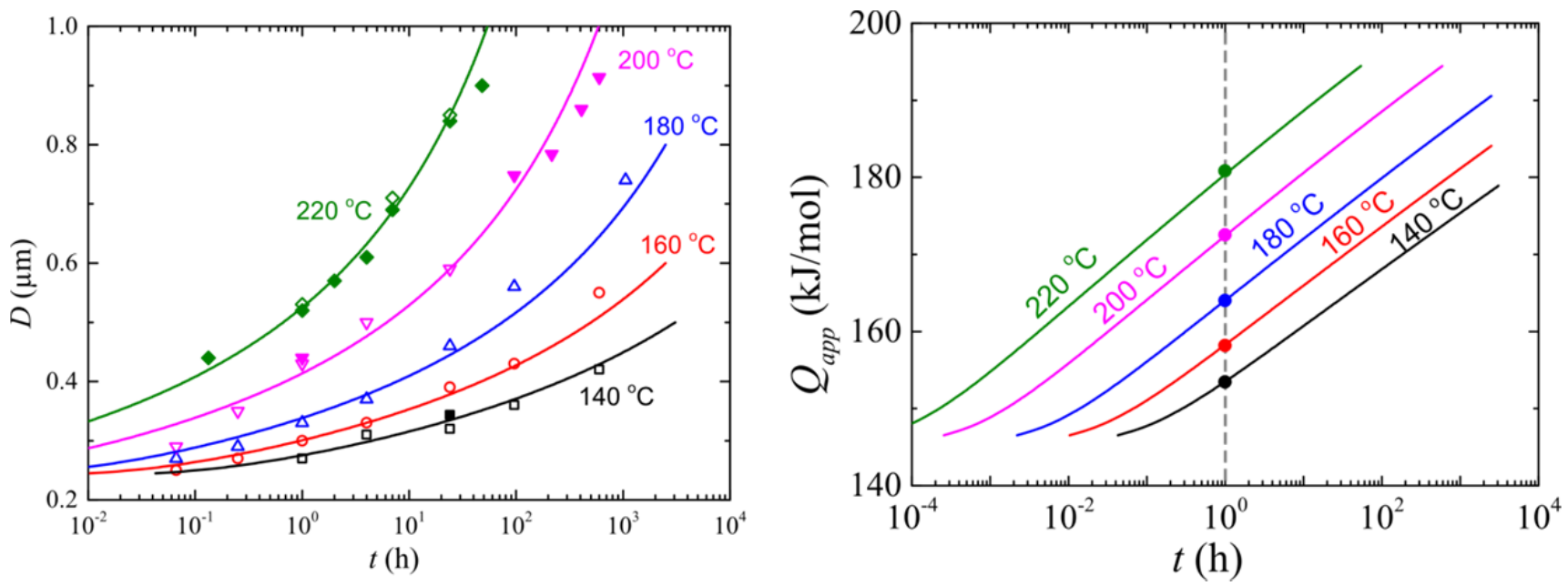